\title{
CONHECIMENTO DE GESTANTES A RESPEITO DE FATORES DE RISCO E PREVENÇÃO DE COMPLICAÇÕES VASCULARES NA GESTAÇÃO
}

\author{
Carolina Ferreira Simões* \\ Arthur Rocha Barros ${ }^{*}$ \\ Dorival Moreschi Junior ${ }^{* *}$
}

\begin{abstract}
RESUM0: Durante o período gestacional, especialmente no segundo trimestre, há um elevado risco de desenvolvimento de eventos tromboembólicos. 0 risco é decorrente de diversos mecanismos adaptativos, que buscam a manutenção da gestação e a viabilidade do feto. Tal risco de complicações se torna maior na presença de trombofilias, distúrbios de coagulação, que são responsáveis por elevado índice de morbimortalidade materna e fetal. A alta taxa demonstra a necessidade de aconselhamento materno, especialmente a respeito de hábitos que devem ser evitados e comportamentos que devem ser praticados durante 0 pré-natal e também no puerpério. Assim, o objetivo desse estudo foi analisar a quantidade de gestantes que conhecem sobre trombofilia na região metropolitana de Maringá - PR. Foram entrevistadas 100 gestantes, a maioria entre 20 a 35 anos de idade. Todas responderam a um mesmo questionário, sendo constatado que $47 \%$ tinham entre 14 e 30 semanas de gestação, $15 \%$ não souberam precisar sua idade gestacional, pois haviam recém-descoberto a gravidez e estavam em sua primeira consulta de pré-natal e $68 \%$ relataram não ter recebido nenhuma informação sobre 0 tema. Apesar da ausência de instruções, uma quantidade siginificativa de participantes respondeu corretamente os questionamentos, sendo significativo o conhecimento em relação à importância da atividade física e da dieta adequada e ao perigo do tabagismo, da obesidade e da presença doenças crônicas, como o diabetes mellitus e a hipertensão arterial sistêmica.
\end{abstract}

PALAVRAS-CHAVE: Gestação; Trombofilias; Complicações Obstétricas; Risco; Prevenção.

\section{INFORMATION OF PREGNANT WOMEN ON RISK FACTORS AND PREVENTION IN VASCULAR COMPLICATIONS DURING PREGNANCY}

\begin{abstract}
There is high risk in developing trombo-embolic occurrences during pregnancy, especially in the second trimester. Risk derives from several adaptative mechanisms that maintain pregnancy and fetus viability. Complication risks are greater in the case of trombophilias or coagulation disorders which cause high levels of maternal and fetal morbo-mortality. High rates reveal the need for counseling especially on habits to be avoided and behavior that should be practiced during the pre-natal and puerperium periods. Current study analyzed the number of pregnant women in the metropolitan region of Maringá, Brazil, that had some information on thrombophilia. One hundred pregnant women, most of them within the 20-35 years bracket, were interviewed. They answered a single questionnaire and provided the following information: 47\% were between the 14th and 30th week of pregnancy; $15 \%$ did not know pregnancy age with any precision since they became recently aware of pregnancy and were making their first visit to the doctor's; $68 \%$ responded that they did not have any information on the subject. In spite of lack of instructions, a significant section of the participants answered the questions correctly, with special reference to physical activity, proper diet, the dangers of smoking, obesity, chronic diseases such as diabetes mellitus and systemic arterial hypertension.
\end{abstract}

KEYWORDS: Pregnancy; Trombophilia; Obstetric Complications; Prevention.

\footnotetext{
Discentes de Medicina no Centro Universitário Cesumar (UNICESUMAR), Maringá (PR), Brasil

* Doutor, Docente no Centro Universitário Cesumar (UNICESUMAR) no Departamento de Medicina; Docente Adjunto do Departamento de Medicina da Universidade Estadual de Maringá (UEM), Maringá (PR), Brasil. E-mail: dr.moreschi@gmail.com
} 


\section{INTRODUÇÃO}

As trombofilias englobam um grupo de desordens da coagulação, que aumentam, potencialmente, a chance de ocorrência de eventos trombóticos, como embolia pulmonar e trombose venosa profunda (TVP), podendo ser hereditárias ou adquiridas (BARROS, 2014).

A presença de tais desordens na gestação é responsável por um elevado índice de morbidade e mortalidade materna e fetal (DAWO0D, 2013).

Durante a gestação, ocorrem inúmeras adaptações fisiológicas que possuem como objetivos o desenvolvimento e a nutrição fetal, a preparação para 0 parto e a prevenção de eventos adversos durante 0 mesmo. Fisiologicamente, as gestantes apresentam estado de hipercoagulabilidade, decorrente da elevação de fatores pró-coagulantes (fatores II, V, VII, VIII, IX, X e XII) e de fibrinogênio, da redução da proteína $S$, resistência à proteína $\mathrm{C}$, maior agregação plaquetária e da diminuição da atividade fibrinolítica. Essas adaptações reduzem os riscos de complicações hemorrágicas no parto (KALIL et al., 2008).

Além de 0 estado de hipercoagulabilidade, durante a gestação ocorre estase venosa. No primeiro trimestre, 0 aumento do fluxo sanguíneo promove elevação da pressão venosa, e nos dois trimestres finais, o útero gravídico realiza compressão da veia cava inferior (KALIL et al., 2008).

Todas essas mudanças no sistema de coagulação elevam o risco de ocorrência de fenômenos troboembólicos e outras alterações vasculares. A presença de trombofilias e a realização de hábitos inadequados incrementam este risco (ALMEIDA, 2011).

Dentre as trombofilias, se observa uma prevalência maior de mutação do Fator $\checkmark$ Leiden (hereditária) e da Síndrome Antifosfolípide (adquirida). A mutação no gene do fator $V$ está relacionada à substituição de glutamina por arginina, na posição 506 do fator e promove interferências na atuação da proteína C, importante anticoagulante. A Síndrome dos Anticorpos Antifosfolípideos (SAAF) é caracterizada pela produção de anticorpos (anticardiolipina e anticoagulante lúpico) que também afetam a cascata de coagulação. As alterações de proteínas $S$ e $C$ podem estar presentes em gestantes, mas com menor prevalência, por serem condições raras; apresentam potencial trombogênico semelhante ao fator $V$ Leiden e a SAAF (LIMA, 2006).

Entre os fatores de risco para trombose em gestantes se destacam as mutações genéticas hereditárias, primiparidade, gestação múltipla, obesidade, tabagismo, hipertensão arterial sistêmica, fertilização in vitro e idade maior do que 35 anos (RADES et al., 2007).

As complicações gestacionais podem ser diversas, como tromboembolismo venoso, préeclâmpsia, abortamentos de repetição, crescimento fetal, parto pré-termo, óbito fetal e/ou materno e descolamento de placenta previamente inserida (RADES et al., 2007).

Não há consenso a respeito do rastreio
para diagnóstico de trombofilias.
Atualmente não se indica rastreio
universal para todas as gestantes.
Trombofilias adquiridas devem ser
investigadas em gestantes com histórico
de três abortos consecutivos precoces ou
uma perda fetal tardia. Já as trombofilias
hereditárias necessitam ser investigadas
em gestantes com histórico pessoal e/
ou familiar (10 grau) de trombofilia ou
de tromboembolismo venoso. Estudos
recentes sugerem não ser necessária a
investigação de trombofilias hereditárias
em pacientes com histórico de
abortamentos de repetição, descolamento
prematuro de placenta e crescimento
intrauterino restrito (LIMA; BORGES 2012).

A investigação para trombofilia não apresenta um consenso estabelecido, por isto deve ser norteada por observações clínicas, históra obstétrica, eventos tromboembólicos prévios, história familiar e 0 fator 
causal da trombofilia (KUPERMAN et al., 2011).

É importante ainda investigar 0 uso de medicações, como anticoagulantes orais, contraceptivos orais, heparina e realização de terapêutica hormonal, visto que essas medicações podem influenciar na interpretação dos exames (FIGUEIRÓ-FILHO, 2012).

A prevenção de complicações vasculares se dá através de uma abordagem terapêutica específica, tendo por base 0 histórico familiar e pessoal e a presença de fatores de risco (KHALAFALLAH, 2014).

A prevenção de eventos adversos, principalmente de trombose venosa profunda, também é feita por mudanças comportamentais. É indicado utilizar meias de compressão, realizar atividade física que envolva principalmente a musculatura dos membros inferiores, não fumar, não ganhar peso excessivamente, realizar dieta adequada, evitar imobilizações por período prolongado e repousar com os pés para cima, para facilitar o retorno venoso (LIMA; BORGES, 2012).

Não encontramos nenhum estudo que avaliasse 0 nível de conhecimento das gestantes sobre esta doença, por isto o objetivo desse estudo foi analisar a quantidade de gestantes que conhecem sobre trombofilia na região metropolitana de Maringá - PR, além do conhecimento das mesmas acerca de ações que auxiliem na prevenção de tais eventos.

\section{MATERIAIS E MÉTODOS}

\subsection{AMOSTRA}

Foram avaliadas 100 gestantes, da região metropolitana de Maringá - PR, entre 0 período de maio e julho de 2015, participantes do pré-natal ou consulta médica geral, nas Unidades Básicas de Saúde Aclimação, Alvorada, Cidade Alta, Céu Azul, Tuiuti e Zona Sul. A seleção das participantes foi realizada de maneira aleatória.

A amostra foi escolhida com base no número de gestantes residentes em Maringá (aproximadamente
4.000) e na prevalência de gestantes acometidas por trombofilias (variação entre 2,5\% e 5\%), dados obtidos no DATASUS.

\subsection{ASPECTOS ÉTICOS}

0 estudo foi aprovado pelo Comitê de Ética em Pesquisa em Seres Humanos do UniCesumar sob o $\mathrm{n}$. 1.067 .910 de 14/05/2015 e as voluntárias assinaram o Termo de Consentimento Livre Esclarecido (TCLE).

\subsection{INTRUMENTO DE PESQUISA E PROCEDIMENTOS}

0 instrumento de pesquisa foi constituído por um questionário, contendo dez questões objetivas e aplicado mediante instruções dos pesquisadores. As questões englobaram informações como: idade, quantidade de filhos, realização de pré-natal, presença de histórico pessoal ou familiar de trombose venosa profunda, complicações obstétricas anteriores e fatores predisponentes e preventivos de complicações vasculares na gestação.

Inicialmente foi explicado para as pacientes 0 objetivo do estudo e assegurado quanto ao anonimato e sigilo das respostas, além de deixar claro 0 caráter voluntário da participação na pesquisa. 0 questionário foi aplicado nas Unidades Básicas de Saúde citadas, durante 0 período antecedente à consulta.

\subsection{ANÁLISE ESTATÍSTICA E CÁLCULOS MATEMÁTICOS}

Foi realizada uma estatística descritiva com média e desvio padrão das idades das pacientes e foi realizado 0 cálculo percentual dos demais itens avaliados, sendo utilizados os softwares Microsoft

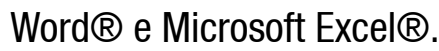

\section{RESULTADOS}

A pesquisa envolveu 100 gestantes, da região metropolitana de Maringá - PR, entre as faixas etárias de 13 a 45 anos de idade (Tabela 1), sendo que $84 \%$ possuíam entre 20 e 35 anos. Em relação à idade gestacional, $47 \%$ tinham entre 14 e 30 semanas de 
gestação (Tabela 2). Um número elevado de gestantes (15\%) não soube precisar sua idade gestacional, pois havia recém-descoberto a gravidez e estava em sua primeira consulta de pré-natal.

Tabela 1. Porcentagem de participantes distribuídas de acordo com a faixa etária $(n=100)$

\begin{tabular}{ccc}
\hline Idade & Frequência $(\mathbf{n})$ & $\%$ \\
\hline$<20$ anos & 9 & $9 \%$ \\
20 e 24 anos & 35 & $35 \%$ \\
25 e 29 anos & 24 & $24 \%$ \\
30 e 35 anos & 25 & $25 \%$ \\
$>35$ anos & 7 & $7 \%$ \\
\hline
\end{tabular}

Tabela 2. Porcentagem de participantes de acordo com a semana de gestação $(n=100)$

\begin{tabular}{ccc}
\hline Semana de gestação & Frequência $(\mathbf{n})$ & $\%$ \\
\hline$\leq 13$ semanas & 20 & $20 \%$ \\
$\geq 14$ semanas e $\leq 30$ semanas & 47 & $47 \%$ \\
$\geq 31$ semanas & 18 & $18 \%$ \\
Não soube precisar & 15 & $15 \%$ \\
\hline
\end{tabular}

Considerando todas as entrevistadas, $91 \%$ afirmou estar participando regularmente de todas as consultas preconizadas durante 0 período pré-natal, enquanto que $9 \%$ relataram participação irregular ou ausência de participação. As gestantes foram questionadas sobre 0 recebimento de informações pelos profissionais de saúde. Com relação a Tabela 3.

Tabela 3. Questionamentos sobre fatores predisponentes de eventos vasculares $(n=100)$

\begin{tabular}{ccc}
\hline Fatores de risco & $\%$ & Frequência (n) \\
\hline Histórico familiar ou pessoal de trombose & $59 \%$ & 59 \\
Tabagismo & $50 \%$ & 50 \\
Obesidade & $63 \%$ & 63 \\
Hipertensão arterial sistêmica & $40 \%$ & 40 \\
Diabetes mellitus & $33 \%$ & 33 \\
Idade maior que 35 anos & $23 \%$ & 23 \\
Repouso prolongado & $14 \%$ & 14 \\
Cirurgias prévias & $9 \%$ & 9 \\
\hline
\end{tabular}


A Tabela 4 apresenta o percentual (\%) e a frequência (n) de possíveis desfechos do desenvolvimento de trombofilias.

Tabela 4. Complicações das trombofilias na gestação e percentual de resposta $(n=100)$

\begin{tabular}{ccc}
\hline Complicações & $\%$ & Frequência (n) \\
\hline Parto prematuro & $50 \%$ & 50 \\
Aborto & $37 \%$ & 37 \\
Pré-eclâmpsia & $32 \%$ & 32 \\
Crescimento intrauterino restrito & $20 \%$ & 20 \\
Descolamento prematuro de placenta & $17 \%$ & 17 \\
Nenhuma das alternativas & $19 \%$ & 19 \\
\hline
\end{tabular}

0 conhecimento sobre possíveis fatores preventivos na prevenção de eventos tromboembólicos são relatados na Tabela 5.

Tabela 5. Questionamentos sobre fatores preventivos de eventos vasculares $(n=100)$

\begin{tabular}{ccc}
\hline Fatores preventivos & $\%$ & Frequência (n) \\
\hline Dieta controlada, conforme orientações médicas & $75 \%$ & 75 \\
Atividade física & $72 \%$ & 72 \\
Deambulação & $54 \%$ & 54 \\
Pernas elevadas & $49 \%$ & 49 \\
Meias de compressão & $36 \%$ & 36 \\
Repouso prolongado & $20 \%$ & 20 \\
Uso de sapato de salto alto & $10 \%$ & \multicolumn{2}{c}{10} \\
Tabagismo & $7 \%$ & \multicolumn{2}{c}{7} \\
Dieta descontrolada, sem preocupação com o ganho de peso & $5 \%$ & \multicolumn{2}{c}{5} \\
Nenhuma das alternativas & $3 \%$ & 3 \\
\hline
\end{tabular}

\section{DISCUSSÃO}

0 presente estudo objetivou analisar a quantidade de gestantes que tinham conhecimento sobre trombofilia, sendo verificado que um percentual muito pequeno de gestantes havia recebido orientação sobre possiveis eventos tromboembólicos na gestação, mesmo assim muitas tinham conhecimento dos possíveis efeitos deste doença no desfecho da mesma e tinham conhecimento dos fatores preventivos para eventos vasculares.
Diversos estudos identificaram prevalência significante de trombose venosa profunda (TVP) identificaram prevalência significante de trombose venosa profunda (TVP) e embolia pulmonar na gravidez. Em pesquisa realizada entre 2004 e 2006 (KALIL et al., 2008) foram registrados 42 casos de tromboembolismo venoso (TEV), de um total de 24.437 gestações. Do total de casos, 26 pacientes desenvolveram TVP por alterações exclusivas da gravidez, enquanto que 12 gestantes apresentaram exames indicativos de trombofilia. 
Dentre as trombofilias, observou-se maior frequência da mutação do fator $V$ de Leiden $(14,2 \%)$ e da Síndrome de Anticorpo antifosfolípide (SAAF) (7,1\%). Os resultados foram semelhantes ao observado por Fonseca (2012), a qual constatou presença de mutação do fator $\mathrm{V}$ de Leiden em $40 \%$ das gestantes com episódio de TVP.

A Sociedade Brasileira de Angiologia e Cirurgia Vascular (SBAVC) considera a presença de trombofilias como condição de alto risco gestacional, em razão de seu início silencioso e de seu alto índice de morbimortalidade materna e fetal, podendo estar presente em $15 \%$ das gestações em que ocorre episódio de tromboembolismo venoso (DUQUE, 2003).

A alta taxa de mortes decorrentes da instalação de tromboembolismo venoso indica a necessidade de conhecimento a respeito do assunto. Pereira et al. (2007) investigaram 40 profissionais de saúde, que oferecem atendimento pré-natal em 20 Unidades Básicas e constataram que $67,5 \%$ declararam-se aptos para 0 fornecimento de orientações quanto à prevenção e controle da doença. Este número é contrastante com o presente estudo, em que $68 \%$ das entrevistadas afirmaram não receber qualquer tipo de orientação sobre 0 tema.

Em relação aos possíveis fatores predisponentes de eventos vasculares na gestação, James et al. (2006) observaram que o principal fator de risco gestacional é a presença de histórico familiar ou pessoal de trombose (OR 24,8). Fonseca (2012) afirma que $25 \%$ dos episódios de trombose venosa profunda são recorrentes. A taxa de recorrência é quatro vezes maior durante 0 período gestacional. Outros fatores, como obesidade (OD 4,4), hipertensão no período gestacional $(\mathrm{OR} 3,8)$ e diabetes mellitus $(\mathrm{OR} 4,0)$ também receberam destaque. No presente estudo, as participantes deram grande enfoque para a obesidade (60\%) e para o histórico pessoal/familiar de TVP (59\%). Em pesquisa realizada por Kalil et al. (2008) foi constatada uma porcentagem $(9,5 \%)$ relevante de casos associados com a realização de cesariana. Ginsberge (2000) destaca que a incidência de TEP é quatro vezes maior em partos cesarianos, quando comparados ao parto normal. No presente estudo, apenas nove gestantes afirmaram que a realização de cirurgias é fator de risco para eventos vasculares, 0 que aponta para uma provável falta de conhecimento sobre os perigos de tal procedimento. No entanto, as participantes demonstraram entendimento em relação à importância do histórico pessoal ou familiar de TVP.

A respeito das possíveis complicações gestacionais, inúmeros estudos validaram a associação das trombofilias com eventos adversos na gestação, tais como abortos de repetição, restrição de crescimento fetal, parto pré-termo, crescimento intrauterino restrito e descolamento prematuro de placenta (KUPFERMINC et al., 1999; MARTINELLI et al., 2001; HARTEL et al., 2005). Em estudo de caso-controle, realizado com 110 gestantes, em que apresentaram complicações obstétricas, 52\% apresentaram trombofilia hereditária (KUPFERMINC et al., 1999). Segundo revisão sistemática, com metanálise, realizada por Robertson et al. (2006), os desfechos que ocorrem com maior frequência foram o parto prematuro, pré-eclâmpsia e a restrição de crescimento fetal. As complicações condizem com os resultados obtidos no presente estudo, em que $50 \%$ das gestantes acreditaram haver risco de prematuridade e mais de $32 \%$ consideraram a possibilidade de ocorrência de pré-eclâmpsia e de aborto (Tabela 4).

Dentre os hábitos recomendados para prevenção de eventos tromboembólicos, Kingman (2003) ressalta a importância de evitar 0 repouso prolongando, especialmente em longas viagens. Robinson et al. (2005) também destacam a deambulação, 0 ato de manter as pernas elevadas e 0 uso de meias elásticas de compressão, como medidas profiláticas de grande relevância. No presente estudo, as participantes evidenciaram a dieta controlada $(75 \%)$ e a realização de atividade física (72\%). 0 hábito de movimentar-se foi assinalado por $54 \%$ das gestantes, 0 que demonstra bom conhecimento sobre tal orientação. Apenas 36\% afirmaram que 0 uso de meias elásticas pode ser benéfico, sugerindo a provável falta de entendimento a respeito de seus benefícios (Tabela 5). 
Para melhor o panorama de conhecimento das gestantes, deve-se buscar fornecer mais orientação sobre 0 tema especialmente para aquelas que estão iniciando o pré-natal ou que estão à espera de seu primeiro filho. Apesar da incidência de trombofilia ser menor do que de outras patologias na gestação, 0 alto elevado número de mortes maternas e fetais indica a imprescindibilidade de divulgação de informações, com enfoque para medidas profilátias que devem ser estimuladas.

Embora existam vários vieses neste tipo de pesquisa e a mesma pode ser estendida para um número maior de gestantes, as conclusões a seguir mostram certa preocupação em relação ao conhecimento desta doença.

\section{CONCLUSÕES}

A partir desse estudo percebe-se que, apesar da falta de divulgação de informações por parte dos profissionais de saúde, a maioria das gestantes possui conhecimento sobre práticas que necessitam ser evitadas ou estimuladas durante o período gestacional, a fim de se evitar a ocorrência de eventos vasculares e que, na presença de um tromboembolismo venoso, há risco de parto prematuro, prê-eclâmpsia e até mesmo de aborto. Ainda assim, um número importante de gestantes (19\%) desconhece a possibilidade de possíveis complicações.

Condições como obesidade, tabagismo, doenças crônicas e histórico pessoal/familiar de trombose venosa profunda são corretamente reconhecidas como fatores de risco por mais da metade das entrevistadas. Um dado interessante é a falta de conscientização sobre os riscos da realização de cirurgias, especialmente do parto cesariano.

Quanto às medidas preventivas, evidenciouse um importante conhecimento em relação à alimentação e atividade física, hábitos destacados por mais de $70 \%$ da amostra. No entanto, foi encontrado um número significativo de gestantes que, erroneamente, acreditam que o repouso prolongado (20\%) seja uma medida profilática.

\section{REFERÊNCIAS}

ALMEIDA, J. M. F. de. Trombofilia Hereditária e Gravidez: controvérsias Actuais. 2010.52f. Dissertação (Mestrado em Integrado em Medicina) - Faculdade de Medicina da Universidade do Porto, 2010.

BARROS, V. I. P. V. L. et al. Resultados gestacionais e trombofilias em mulheres com histórico de óbito fetal de repetição. Revista Brasileira de Ginecologia e Obstetrícia, v. 36, n. 2, p. 50-55, 2014.

DAW00D, F. Pregnancy and thrombophilia. Journal Blood Disorders and Transfusion, v. 4, n. 164, p. 2, 2013.

DUQUE, F. L.V.; MELLO, N. A. Trombogênesetrombofilia. Journal of Vascular Surgery, v. 2, n. 2, p. 105-118, 2003.

FIGUEIRÓ-FILHO, E. A. Marcadores séricos de trombofilias hereditárias e anticorpos antifosfolípides em gestantes com antecedentes de pré-eclâmpsia grave. Revista Brasileira de Ginecologia e Obstetrícia, v. 34, n. 1, 2012.

FONSECA, A. G. As Trombofilias Hereditárias na Grávida: do Risco Trombótico ao Sucesso da Gravidez. Acta Medica Portuguesa, v. 25, n. 6, 2012.

GINSBERG, J. S.; BATES, S. M. Safety profile of different low-molecular weight heparins used at therapeutic dose. Haematologica, v. 85, p. 935-42, 2000.

HÄRTEL, C. et al. Polymorphisms of haemostasis genes as risk factors for preterm delivery. Thrombosis and Haemostasis, v. 94, n. 1, p. 88-92, 2005.

JAMES, A. H. et al. Venous thromboembolism during pregnancy and the postpartum period: incidence, risk factors, and mortality. American Journal of Obstetrics and Gynecology, v. 194, n. 5, p. 1311-1315, 2006. 
KALIL, J. A. et al. Investigação da trombose venosa na gravidez. Jornal Vascular Brasileiro, v. 7, n. 1, p. 2837, 2008.

KHALAFALLAH, A. A. et al. Review of Management and Outcomes in Women with Thrombophilia Risk during Pregnancy at a Single Institution. ISRN Obstetrics and Gynecology, v. 2014, 2014.

KINGMAN, C.E.; ECONOMIDES, D. L. Travel in pregnancy: pregnant women's experiences and knowledge of health issues. Journal of Travel Medicine, v. 10, n. 6, p. 330-333, 2003.

KUPFERMINC, M. J. et al. Increased frequency of genetic thrombophilia in women with complications of pregnancy. New England Journal of Medicine, v. 340 , n. 1, p. 9-13, 1999.

KUPERMAN, A.; SARIG, G.; BRENNER, B. New scoring system for estimating the risk for pregnancy complications and venous thromboembolism in women with thrombophilia. Harefyah, v. 150, n. 4, p. 363-368, abr. 2011.

LIMA, J. Trombofilias e gravidez. Boletim da Sociedade Portuguesa de Hemorreologia e Microcirculação, v. 21, n. 5, p. 6-23, 2006.

LIMA, J.; BORGES, A. Rastreio de trombofilias. Boletim da Sociedade Portuguesa de Hemorreologia e Microcirculação, v. 27, n. 4, p. 5-11, 2012.

LOCKWOOD, C.; WENDEL, G. Practice bulletin no. 124: inherited thrombophilias in pregnancy. Obstetrics and Gynecology, v. 118, n. 3, p. 730-740, 2011.

PEREIRA DA COSTA, F. L. et al. Trombose venosa profunda na gestação: conhecimento e prática profissional. Enfermería Global, v. 6, n. 10, 2007.

RADES,É. etal. Trombofilias hereditárias e repercussões fetais e perinatais: 0 que há de novo? Femina, v. 35, n. 9, p. 573-578, 2007.

ROBERTSON, L. et al. Thrombophilia in pregnancy: a systematic review. British Journal of Haematology, v. 132, n. 2, p. 171-196, 2006.

ROBINSON, S. et al. The management and outcome of 18 pregnancies in women with polycythemia vera. Haematologica, v. 90, n. 11, p. 1477-1483, 2005.

SILVEIRA, P. R. M. da. Trombose venosa profunda e gestação: aspectos etiopatogênicos e terapêuticos. Jornal Vascular Brasileiro, v. 1, n. 1, p. 65-70, 2002.

Recebido em: 25 de maio de 2016 Aceito em: 06 de junho de 2016 